1 MECHANICS OF ADVANCED MATERIALS AND STRUCTURES

\title{
2 Static and dynamic analysis of homogeneous Micropolar-Cosserat 3 panels
}

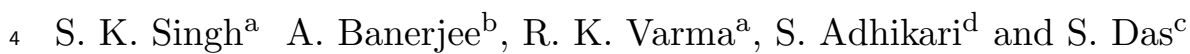

5 a Department of Civil Engineering, Indian Institute of Technology Jammu, Jagti, Nagrota, 6 NH-44, India; ${ }^{\mathrm{b}}$ Department of Civil Engineering, Indian Institute of Technology Delhi, Hauz 7 Khas, 110016, India; ' Department of Civil Engineering,University of Windsor, 401 Sunset 8 Avenue, Canada; ${ }^{\mathrm{d}}$ Zienkiewicz Centre for Computational Engineering, Swansea University, 9 Swansea SA1 8EN, UK

\author{
ARTICLE HISTORY \\ Compiled February 24, 2021
}

\section{ABSTRACT}

This paper communicates an analytical study on computing the natural frequencies and in-plane deflections caused by static forces in the panel walls using EulerBernoulli, Timoshenko, Timoshenko and Goodier, Couple-stress, and MicropolarCosserat theory. The study highlights the formulation of the transfer matrix via the state-space method in the spatial domain; from coupled governing equations of motion that arises from the Micropolar-Cosserat theory. This theory captures the novel curvature of edges and moments of the panels at energy density level due to its unique feature of asymmetric shear stresses; that emphasizes the loss of ellipticity of governing equations. The analytical solution of the Micropolar-Cosserat theory yield appropriate results compared to plane-stress simulation of the panels using finite element analysis.

\section{KEYWORDS}

Couple-stress theory; Micropolar-Cosserat panel; size-dependent behavior; eigenvalue problems.

\section{Table of symbols}

We summarize a reference table of symbols and descriptions used in the paper.

CONTACT. Tel: +44 (0)1792 602088, Fax: + $44(0) 1792295676$

S. K. Singh. Email: 2018rce0002@iitjammu.ac.in

A. Banerjee. Email: abanerjee@iitd.ac.in

R. K. Varma. Email: rajendra.varma@iitjammu.ac.in

S. Adhikari. Email: S.Adhikari@swansea.ac.uk

S. Das. Email: sdas@uwindsor.ca 


\begin{tabular}{ccc}
\hline S.N. & Symbols & Description \\
\hline \hline 1 & $L$ & Length \\
2 & $W$ & Width \\
3 & $T$ & Thickness \\
4 & $A$ & Cross-section area \\
5 & $I$ & Second moment of area \\
6 & $\rho$ & Density \\
7 & $\nu$ & Poisson ratio \\
8 & $E$ & Young modulus \\
9 & $G$ & Shear modulus \\
10 & $G_{c}$ & Cosserat modulus \\
11 & $k$ & Shear coefficient \\
12 & $l$ & Characteristics length \\
13 & $u_{x}$ & Longitudinal deflection \\
14 & $u_{y}$ & Transverse deflection \\
15 & $\phi$ & Rotation of cross-section \\
16 & $\psi_{z}$ & Rigid micro-rotation \\
17 & $\psi$ & Independent micro-rotation \\
18 & $\epsilon_{x}, \epsilon_{y}$ & Normal strains \\
19 & $\epsilon_{x y}, \epsilon_{y x}$ & Transverse strains \\
20 & $\gamma_{s}$ & Symmetric shear strain \\
21 & $\gamma_{a}$ & Asymmetric shear strain \\
22 & $K_{x z}, K_{y z}$ & Plane-stress curvatures \\
23 & $\sigma_{x}, \sigma_{y}$ & Normal stresses \\
24 & $\tau_{x y}, \tau_{y x}$ & Shear stresses \\
25 & $m_{x z}, m_{y z}$ & Curvature moments \\
26 & $M_{x}$ & Moment force \\
27 & $Q_{x y}, Q_{y x}$ & Shear Force \\
28 & $P_{x z}$ & Curvature force \\
29 & $\zeta$ & Eigenvector \\
30 & $\Omega$ & Eigenvalue \\
31 & $\omega$ & Forcing frequency \\
32 & $\omega_{n}$ & Natural frequency \\
33 & $N_{f}$ & Normalised frequency \\
\hline & &
\end{tabular}

\section{Introduction}

Due to its lightweight, panel walls are used as an effective alternative to the conventional bricks walls. Infill panel walls provide a degree of thermal insulation, acoustic insulation, weather resistance, improve the appearance of buildings, and support the cladding system (Lawson, M. et al. , 2001); however, it does not carry any static floor load. Panel walls are subjected to the lateral load during an earthquake. In this paper, the in-plane static and dynamic characteristics of a homogeneous panels have been analytically evaluated. Panels are often modeled employing beam or plane stress elements. Current research has focused on developing new mathematical models which consider physical properties of materials at micro and nano-scales (Carrera, E., \& Zozulya, V. V. , 2020). The higher-order beam theories are capable of capturing the curvature of edges at small scale parameters (Carrera, E., \& Zozulya, V. V. et al. , 2019; Asghari, M., Kahrobaiyan, M. H., Rahaeifard, M., \& Ahmadian, M. T. et al. , 
2011). Based on the underlying mechanics beam theories are classified in the following classes:

(1) Euler-Bernoulli beam theory neglects the shear deformation and rotary inertia of the cross-section, which restricts it for thin beams only (Ghugal, Y. M., \& Shimpi, R. P. et al. , 2001). The governing equation of motion for free vibration can be written as:

$$
D_{x} \frac{\partial^{4} u_{y}}{\partial x^{4}}+\rho A \frac{\partial^{2} u_{y}}{\partial t^{2}}=0 .
$$

(2) Lord Rayleigh added the rotary inertia of the cross-section (Elishakoff, I., Kaplunov, J., \& Nolde, E. et al. , 2015; Banerjee, A. et al. , 2020) in the governing equation of the Euler-Bernoulli beam. The free vibration equation can be expressed as:

$$
D_{x} \frac{\partial^{4} u_{y}}{\partial x^{4}}+\rho A \frac{\partial^{2} u_{y}}{\partial t^{2}}-\rho I \frac{\partial^{4} u_{y}}{\partial x^{2} \partial t^{2}}=0 .
$$

(3) Timoshenko added the shear deformation of the cross-section (Elishakoff, I., Kaplunov, J., \& Nolde, E. et al. , 2015) in addition to the Eq. (2). Thus, Timoshenko beam equation can be written as:

$$
\begin{aligned}
D_{x} \frac{\partial^{2} \phi}{\partial x^{2}}-D_{s} \kappa\left(\frac{\partial u_{y}}{\partial x}+\phi\right)-\rho I \frac{\partial^{2} \phi}{\partial t^{2}} & =0 \\
D_{s} \kappa \frac{\partial}{\partial x}\left(\frac{\partial u_{y}}{\partial x}+\phi\right)-\rho A \frac{\partial^{2} u_{y}}{\partial t^{2}} & =0 .
\end{aligned}
$$

(4) In the couple stress theory, axial deformation, two higher-order material length scale parameters, and micro-inertia (Asghari, M., Kahrobaiyan, M. H., Rahaeifard, M., \& Ahmadian, M. T. et al. , 2011) have also been considered in addition to Eq. (3) and equation of motion can be written as:

$$
\begin{array}{r}
D_{l} \frac{\partial^{2} u_{x}}{\partial x^{2}}-\rho A \frac{\partial^{2} u_{x}}{\partial t^{2}}=0 \\
D_{x} \frac{\partial^{2} \phi}{\partial x^{2}}-D_{s} \kappa\left(\frac{\partial u_{y}}{\partial x}+\phi\right)+\frac{D_{x z}}{2} \frac{\partial^{2}}{\partial x^{2}}\left(\phi-\frac{\partial u_{y}}{\partial x}\right)-\rho I \frac{\partial^{2} \phi}{\partial t^{2}} \\
-\frac{\rho A J}{4}\left(\frac{\partial^{2} \phi}{\partial t^{2}}-\frac{\partial^{3} u_{y}}{\partial x \partial t^{2}}\right)=0 \\
D_{s} \kappa \frac{\partial}{\partial x}\left(\frac{\partial u_{y}}{\partial x}+\phi\right)+\frac{D_{x z}}{2} \frac{\partial^{3}}{\partial x^{3}}\left(\phi-\frac{\partial u_{y}}{\partial x}\right)-\rho A \frac{\partial^{2} u_{y}}{\partial t^{2}} \\
-\frac{\rho A J}{4}\left(\frac{\partial^{3} \phi}{\partial x \partial t^{2}}-\frac{\partial^{4} u_{y}}{\partial x^{2} \partial t^{2}}\right)=0 .
\end{array}
$$

where, $D_{l}=E A, D_{x}=E I, D_{s}=G A$ and $D_{x z}=G A l^{2}$ are stiffness parameters; $E, A, I, J, G, \rho, \kappa$, and $l$ represents Young modulus, a cross-sectional area, second moment of area, micro-inertia, shear modulus, density, Timoshenko shear coefficient and characteristics length, respectively. 
Euler-Bernoulli beam theory neglects transverse shear strains and miscarry the deflection and natural frequency in case of thick beams where shear deformation effects are significant (Ghugal, Y. M., \& Shimpi, R. P. et al. , 2001). Rayleigh proposed an improvement to the Euler-Bernoulli beam theory by including the effect of rotary inertia of the cross-section of the beam (Labuschagne, A., van Rensburg, N. J., \& Van der Merwe, A. J. et al. , 2009). Timoshenko proposed his theory where shear deformation of the cross-section is also taken into account (Timoshenko, S. P. , 1921). However, transverse shear strain is ignored in Rayleigh theory (Elishakoff, I., Kaplunov, J., \& Nolde, E. et al. , 2015). These theories are proved very fruitful to both theoretical as well as experimental aspects (Ghugal, Y. M., \& Shimpi, R. P. et al. , 2001). In the classical continuum mechanics, the motion of material particles are described by position vectors identifying the location of each particle as a function of time. (Rubin, M. B. et al. , 2013). So, in the classical theories, every particle has three displacements which are calculated by symmetric stress tensor that is not sufficient for describing the micro and nano-scales size of second-phase particles (Czekanski, A., \& Zozulya, V. V. et al. , 2019; Wu, B., Pagani, A., Chen, W. Q., \& Carrera, E. , 2019; Zozulya, V. V. et al. , 2017). However, in most of the engineering problems; micro and nanoscale structures, the major concern in deformations is inelastic range, and observed that strain gradient effect generally holds the regime (Xue, Z., Huang, Y., \& Li, M. , 2002). It is a significant fact that the size of second-phase particles has an important effect on the macroscopic behaviour of materials (Cao, Y. P., \& Lu, J. et al. , 2005). The strain gradient based theory of elasticity to investigate the particle size effect find good agreements with the experiments as well as numerical studies. The preservation of the planeness of cross-section requires that the averaging length should be larger than the beam depth (Sun, Z. H., Wang, X. X., Soh, A. K., Wu, H. A., \& Wang, Y. , 2007; Karttunen, A. T., Romanoff, J., \& Reddy, J. N. et al. , 2016). The couple stress theory is a non-classical continuum theory based on macro-deformation and micro-rotation in which the full curvature vector is used to calculate the deformation in addition to the conventional strain (Asghari, M., Kahrobaiyan, M. H., Rahaeifard, M., \& Ahmadian, M. T. et al. , 2011; Karttunen, A. T., Romanoff, J., \& Reddy, J. N. et al. , 2016; Sobhy, M., \& Zenkour, A. M. et al. , 2020). So, the mechanical behaviour of structures based on strain gradient is capable of capturing the effect on small-scale particles, when the characteristic size of structures is close to the material length parameter (Carrera, E., \& Zozulya, V. V. et al. , 2019; Chen, W., \& Si, J. 2013; Ebrahimi, F., \& Barati, M. R. et al. , 2018a,b). In the couple stress theory, the rotation of the micro-structure and macro-structure is deemed to be equal and no constitutive equation is written for asymmetric shear stress vector. This vector is determined by considering the micro-structure rotational equation of motion of the elements (Asghari, M., Kahrobaiyan, M. H., Rahaeifard, M., \& Ahmadian, M. T. et al. , 2011; Chen, W., \& Wang, Y. et al. , 2016). Hence, the asymmetric part of the shear stress does not contribute to the energy density (Asghari, M., Kahrobaiyan, M. H., Rahaeifard, M., \& Ahmadian, M. T. et al. , 2011; Karttunen, A. T., Romanoff, J., \& Reddy, J. N. et al. , 2016). Euler-Bernoulli, Rayleigh, Timoshenko, and couple stress theories have been successfully implemented for the analysis of beams and extended for panels (Ventsel, E., Krauthammer, T., \& Carrera, E. J. A. M. R. et al. , 2002). However, these theories lack to predict the behavior such as shear deformation and rotational inertia of cross-section, shear deformation, strain gradient effects, and curvature moment contribution at energy density level, respectively. Infill wall shows the curvature of edges which is not predicted accurately by these theories due to the absence of curvature vector mechanism based on the constitutive relation (Elishakoff, 
I., Kaplunov, J., \& Nolde, E. et al. , 2015; Asghari, M., Kahrobaiyan, M. H., Rahaeifard, M., \& Ahmadian, M. T. et al. , 2011; Toupin, R. A. et al. , 1964; Cosserat, E., \& Cosserat, F. et al. , 1909).

In this present article, Micropolar-Cosserat linear elastic beam theory has been considered to capture the curvature behavior (based on the constitutive relation or at energy density level) of the infill wall with appropriate stiffness parameters (Karttunen, A. T., Reddy, J. N., \& Romanoff, J. et al. , 2018; Ramezani, S., Naghdabadi, R., \& Sohrabpour, S. et al. , 2009). The assumption, and characteristic features of the Micropolar-Cosserat continuum contains micro-structure which can rotate independently from the surrounding medium, and existence of couple stresses and asymmetric shear stresses, respectively (Noor, A. K., \& Nemeth, M. P. , 1980; Ramezani, S., Naghdabadi, R., \& Sohrabpour, S. et al. , 2009; Zozulya, V. V. et al. , 2018). The initial theoretical work was done by the Cosserat brothers (Cosserat, E., \& Cosserat, F. et al. , 1909), Mindlin (Mindlin, R. D. et al. , 1965), and Nowacki (Nowacki, W. et al. , 1972). Eringen (Eringen, A. C. et al. , 1968) explained the micro-inertia which describe the dynamics effects of microstructure. This additional constant micro-rotation field throughout the width of the beam converts the Timoshenko beam theory (first-order shear deformation theory) into Micropolar-Cosserat elastic beam theory (Mindlin, R. D., \& Tiersten, H. F. et al. , 1962; Nowacki, W. et al. , 1974). So, each element of Micropolar-Cosserat continuum have three translational motion and three rotational ones, which are assigned to macro-structures and micro-structures, respectively (Ramezani, S., Naghdabadi, R., \& Sohrabpour, S. et al. , 2009; Eringen, A. C. et al. , 1999). In Micropolar-Cosserat theory, the mutual interaction between two adjacent surface elements is expressed via the traction vector in addition to the couple-stress vector. While, the effect of a surface element on a neighboring one is expressible by a traction vector only; from the kinetic point of view in the classical continuum theory (Ramezani, S., Naghdabadi, R., \& Sohrabpour, S. et al. , 2009; Eringen, A. C. et al. , 2001, 2012). Dugem and Voitgt (Zozulya, V. V. et al. , 2017) suggested that the relationship between two adjacent elements of the body depends on the surface area element; employing force and couple stress vector (Kumar, R., \& Ailawalia, P. et al., 2005; Gharahi, A., \& Schiavone, P. et al. , 2020). However, the complete theory of asymmetric elasticity was developed by the Cosserat brothers (Cosserat, E., \& Cosserat, F. et al. , 1909). The asymmetric elasticity is the unique features of Micropolar-Cosserat theory to distinguish it from other standard theories. The shear stress can be split into symmetric and asymmetric shear stresses which facilitates the full curvature tensor to capture the micro-rotation in addition to the conventional strain (Mindlin, R. D. et al. , 1963; Khoei, A. R., Yadegari, S., \& Biabanaki, S. O. R. et al. , 2010). The symmetric shear stress causes the deformation of macro-structure and asymmetric shear stresses contribute to the rigid rotation of microstructure of the material. Hence, This theory provides the proficient gear to curvature moment at micro-scale (Ramezani, S., Naghdabadi, R., \& Sohrabpour, S. et al. , 2009; Cao, Y. P., \& Lu, J. et al. , 2005).

In this work, a 1-D Micropolar-Cosserat elastic governing equation of motion based on the linear law of variation of displacement has been considered for analysis of panels. Exact in-plane macro and micro displacement, and natural frequency of the panels have been evaluated implementing the transfer matrix approach and the state-space method (Banerjee, A. et al. , 2020; Banerjee, J. R. et al. , 2001; Dion, J. M., \& Commault, C. et al. , 1993). The boundary condition taken by other authors corresponding to the Micropolar-Cosserat elastic continuum; deflection and resultant force is equal to zero at fixed end and free end, respectively (Karttunen, A. T., Romanoff, J., \& 
Reddy, J. N. et al. , 2016; Karttunen, A. T., Reddy, J. N., \& Romanoff, J. et al. , 2018; Ramezani, S., Naghdabadi, R., \& Sohrabpour, S. et al. , 2009). These boundary conditions are not sufficient to have zero value of the curvature moment at free end section. So, it is necessary to have the exact values as a part of the loading definition (Asghari, M., Kahrobaiyan, M. H., Rahaeifard, M., \& Ahmadian, M. T. et al. , 2011; Augarde, C. E., \& Deeks, A. J. et al. , 2008). In the present paper, the curvature moment (or force) has been considered due to asymmetric shear at free end to find the in-plane static exact response. Another unique feature of this work is a validation of theoretical independent micro-rotation of panel with the help of static response of the plane-stress element. Moreover, the study of various beam theories like; Euler-Bernoulli, Timoshenko, Timoshenko and Goodier exact analysis, Couple stress theory, and their comparison with analytical v/s finite elements analysis have done for building confidence. The proposed methodology can be extended for the composite and functionally graded panels very effectively, but for the brevity and develop the insight on the theory, this paper is limited only for the analysis of homogeneous panels (Hoffman, R. E., \& Ariman, T. et al. , 1968; Vasiliev, V. V., Barynin, V. A., \& Rasin, A. F. et al. , 2001; Reddy, J. N. et al. , 2011).

\section{Micropolar-Cosserat elastic panel theory}

\subsection{Two-dimensional equilibrium equations}

The Micropolar-Cosserat solid can transmit normal as well as bending stresses due to having an extra macro-rotational degree of freedom. The sketch shown in Fig. 1 depicts a 2-D free body diagram of the typical Micropolar-Cosserat element associated with the varying stress field.

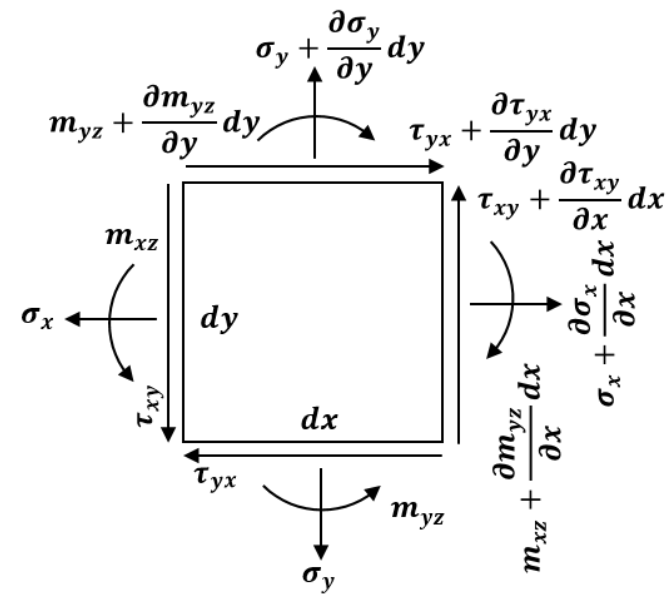

(a)

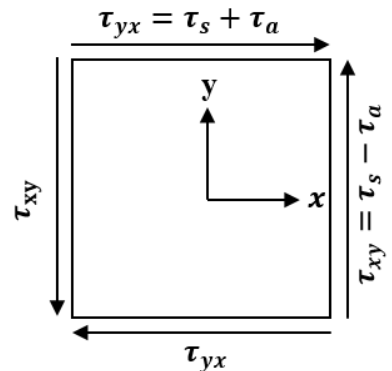

( b )

Figure 1. (a) Normal and bending stresses acting on a planar Micropolar-Cosserat solid in a varying stress field, and (b) The symmetric and asymmetric parts of the shear stresses.

The plane-stress equilibrium equations of motion for Micropolar-Cosserat element 
are written as follows

$$
\frac{\partial \sigma_{x}}{\partial x}+\frac{\partial \tau_{y x}}{\partial y}-\rho \frac{\partial^{2} u_{x}}{\partial t^{2}}=0
$$

189

$$
\frac{\partial \sigma_{y}}{\partial y}+\frac{\partial \tau_{x y}}{\partial x}-\rho \frac{\partial^{2} u_{y}}{\partial t^{2}}=0
$$

190

$$
\frac{\partial m_{x z}}{\partial x}+\frac{\partial m_{y z}}{\partial y}+\left(\tau_{y x}-\tau_{x y}\right)-\rho J \frac{\partial^{2} \psi_{z}}{\partial t^{2}}=0,
$$

Unlike in the Micropolar-Cosserat theory, an additional equilibrium equation for the curvature moment does not appear in the modified couple stress theory (Karttunen, A. T., Reddy, J. N., \& Romanoff, J. et al. , 2018; Park, S. K., \& Gao, X. L. et al. , 2008). It can be seen from Eq. (7) that the shear stresses are not necessarily symmetric, which is the unique features of the Micropolar-Cosserat theory to distinguish it from other standard theory (Lam, D. C., Yang, F., Chong, A. C. M., Wang, J., \& Tong, P. et al. , 2003).

\subsection{Stress-strain of Micropolar-Cosserat panel}

The positive directions of the stress resultants, displacements, and cross-sectional shape of the panel after the development of force and couple stresses are shown in Fig. 2.

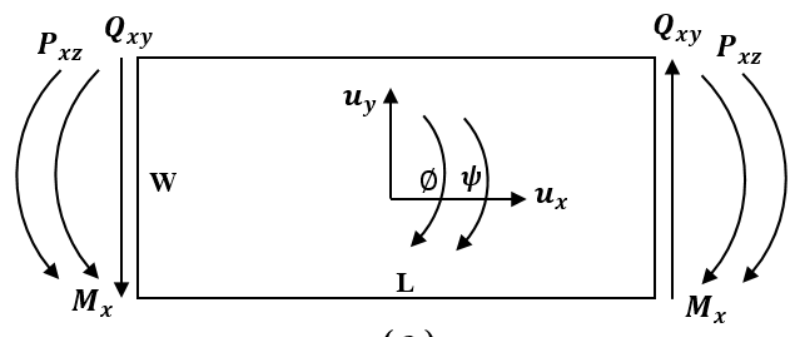

( a )

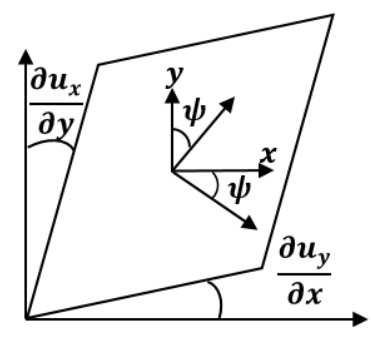

( b )

Figure 2. (a) Micropolar-Cosserat elastic panel and (b) Relative strains and rigid rotation of micro-structure.

Let us consider a 2-D homogeneous, isotropic and linear elastic panel of a length $L$ with rectangular cross-section of constant width $W$, and thickness $T$. The equations of displacement field based on the linear law of variation are

$$
u_{x}(x, y, t)=y \phi(x, t), u_{y}(x, y, t)=u_{y}(x, t), \text { and } \psi_{z}(x, y, t)=\psi(x, t) .
$$

The normal strains are

$$
\epsilon_{x}=\frac{\partial u_{x}}{\partial x}=y \phi^{\prime}, \text { and } \epsilon_{y}=\frac{\partial u_{y}}{\partial y}=0 .
$$


The relatives asymmetric shear strains are

$$
\epsilon_{x y}=\left(\frac{\partial u_{x}}{\partial y}-\psi_{z}\right)=(\phi-\psi), \text { and } \epsilon_{y x}=\left(\frac{\partial u_{y}}{\partial x}+\psi_{z}\right)=\left(u_{y}^{\prime}+\psi\right) .
$$

Where $u_{x}, u_{y}, \phi, \psi_{z}$, and $\psi$ are the longitudinal, transverse, rotation of the crosssection about the neutral axis of the panel, rigid micro-rotation, and an independent micro-rotation of micro-structure respectively. In the microstructures, the rotating axis is called orthogonal directors and directors of each material point are deformable in the Micropolar-Cosserat solid (Karttunen, A. T., Reddy, J. N., \& Romanoff, J. et al. , 2018; Reddy, J. N. , 2003). The symmetric and skew-symmetric shear strains are defined, respectively as

$$
\gamma_{s}=\left(u_{y}^{\prime}+\phi\right), \text { and } \gamma_{a}=\left(u_{y}^{\prime}-\phi+2 \psi\right) .
$$

We can see that the symmetric part takes the same form as the shear strain in the classical Timoshenko beam theory. The skew-symmetric part is twice the difference between the usual macro-rotation and the micro-rotation (Eringen, A. C. et al. , 2012). The curvatures describe the bending of planer elements due to couple-stresses are

$$
K_{x z}=\frac{\partial \psi_{z}}{\partial x}, \text { and } K_{y z}=\frac{\partial \psi_{z}}{\partial y}=0 .
$$

The localization of shear deformation at the material length scale parameter has been quantified thus enabling both the Cosserat modulus and characteristic length as an additional constitutive parameter present into the Micropolar-Cosserat continuum (De Borst, R., \& Sluys, L. J. et al. , 1991; De Borst, R. E. N. É. et al , 1991). The isotropic stress-strain relationship for one-dimensional Micropolar-Cosserat panel can be written as

$$
\left\{\begin{array}{c}
\sigma_{x} \\
\tau_{x y} \\
\tau_{y x} \\
m_{x z}
\end{array}\right\}=\left[\begin{array}{cccc}
E & 0 & 0 & 0 \\
0 & G+G_{c} & G-G_{c} & 0 \\
0 & G-G_{c} & G+G_{c} & 0 \\
0 & 0 & 0 & 2 G l^{2}
\end{array}\right]\left\{\begin{array}{c}
\epsilon_{x} \\
\epsilon_{x y} \\
\epsilon_{y x} \\
K_{x z}
\end{array}\right\}
$$

where $G_{c}$ represents Cosserat modulus of the homogeneous panel.

The characteristics length represents a material property and order of the magnitude as the maximum size of material inhomogeneities with solely softening yield (Tran, T. H., Monchiet, V., \& Bonnet, G. et al. , 2012). However, plastic nature also developing during severe deformation of ductile materials with softening followed by hardening (Tordesillas, A., Peters, J. F., \& Gardiner, B. S. et al. , 2004). In Micropolar-Cosserat continuum analysis, the numerical value proposed for $G_{c}$ is $\frac{G}{3}$ and ratio of $\frac{l}{L}$ are 0.02083 , for static and 0.01042 , for dynamic analysis. In couple stress analysis, the numerical value of $G_{c}$ is neglected and ratio of $\frac{l}{L}$ are 0.02083 , for static and 0.01042 , for dynamic analysis (De Borst, R., \& Sluys, L. J. et al. , 1991; De Borst, R. E. N. É. et al , 1991; Asghari, M., Rahaeifard, M., Kahrobaiyan, M. H., \& Ahmadian, M. T. et al. , 2011; Hadjesfandiari, A. R., Hajesfandiari, A., Zhang, H., \& Dargush, G. F. et al. , 2017; Khoei, A. R., \& Karimi, K. et al. , 2008). 


\subsection{Balance equations for Micropolar-Cosserat panel}

Let us consider stress and displacement field do not vary across the width. Stress-strain and couple stress components are independent of z-coordinate. The elastic constants are the function of $\mathrm{x}$-coordinate. The applied load is so that, no torsion occurs in the beam (Karttunen, A. T., Reddy, J. N., \& Romanoff, J. et al. , 2018; Ramezani, S., Naghdabadi, R., \& Sohrabpour, S. et al. , 2009). The balanced equations for 1-D Micropolar-Cosserat panel are expressed as

$$
\frac{\partial M_{x}}{\partial x}-Q_{y x}-\rho \frac{\partial^{2} U_{x}}{\partial t^{2}}=0
$$

$$
\frac{\partial Q_{x y}}{\partial x}-\rho A \frac{\partial^{2} u_{y}}{\partial t^{2}}=0
$$

$$
\frac{\partial P_{x z}}{\partial x}+\left(Q_{y x}-Q_{x y}\right)-\rho A J \frac{\partial^{2} \psi_{z}}{\partial t^{2}}=0
$$

where

$U_{x}=\int_{A} u_{x} y d A$ and $J$, cubical element $=\frac{2 l^{2}}{1+\nu}($ DeBorst, R., \&Sluys, L.J.etal., 1991).

The stress resultants to reduce the 2-D equilibrium equations into 1-D balanced equations are as follows

$$
M_{x}=\int_{A} \sigma_{x} y d A, Q_{x y}=\int_{A} \tau_{x y} d A, Q_{y x}=\int_{A} \tau_{y x} d A, \text { and } P_{x z}=\int_{A} m_{x z} d A .
$$

From the isotropic stress-strain relationship Eq. (13) and stress resultants Eq. (17), following can be expressed as

$$
\sigma_{x}=E \epsilon_{x}=E y \phi^{\prime}
$$

$$
\begin{gathered}
M_{x}=E I \phi^{\prime}=D_{x} \phi^{\prime}, \\
\tau_{x y}=\left(G+G_{c}\right) \epsilon_{x y}+\left(G-G_{c}\right) \epsilon_{y x}
\end{gathered}
$$

$$
\begin{gathered}
Q_{x y}=D_{s}\left(u_{y}^{\prime}+\phi\right)-D_{a}\left(u_{y}^{\prime}-\phi+2 \psi\right), \\
\tau_{y x}=\left(G-G_{c}\right) \epsilon_{x y}+\left(G+G_{c}\right) \epsilon_{y x}
\end{gathered}
$$

$$
Q_{y x}=D_{s}\left(u_{y}^{\prime}+\phi\right)+D_{a}\left(u_{y}^{\prime}-\phi+2 \psi\right),
$$




$$
m_{x z}=2 G l^{2} K_{x z}=2 G l^{2} \psi^{\prime}
$$

251

$$
P_{x z}=2 G l^{2} A \frac{\partial \psi}{\partial x}=2 D_{x z} \psi^{\prime},
$$

265

266

where $D_{a}=G_{c} A$ is the Cosserat stiffness parameter for a homogeneous panel. The stiffness parameters can also be helpful to represent, a functionally graded material (Reddy, J. N. et al. , 2011; Reddy, J. N. , 2003).

\subsection{Governing equations of motion}

\subsubsection{Dynamic system}

Governing equations of motion for 1-D Micropolar-Cosserat panel are derived by substituting the value of stress and force resultant Eqs. (18) to (21) into balance Eqs. (14) to (16). They are as follows

$$
D_{s}\left(u_{y}^{\prime \prime}+\phi^{\prime}\right)-D_{a}\left(u_{y}^{\prime \prime}-\phi^{\prime}+2 \psi^{\prime}\right)-\rho A \frac{\partial^{2} u_{y}}{\partial t^{2}}=0
$$

$$
D_{x} \phi^{\prime \prime}-D_{s}\left(u_{y}^{\prime}+\phi\right)-D_{a}\left(u_{y}^{\prime}-\phi+2 \psi\right)-\rho I \frac{\partial^{2} \phi}{\partial t^{2}}=0
$$

$$
2 D_{x z} \psi^{\prime \prime}+2 D_{a}\left(u_{y}^{\prime}-\phi+2 \psi\right)-\rho A J \frac{\partial^{2} \psi}{\partial t^{2}}=0
$$

\subsubsection{Static system}

By substituting time-dependent macro and micro displacement is equal to zero into the dynamic system of Eqs. (22) to (24), the equations derived are as follows

$$
D_{s}\left(u_{y}^{\prime \prime}+\phi^{\prime}\right)-D_{a}\left(u_{y}^{\prime \prime}-\phi^{\prime}+2 \psi^{\prime}\right)=0,
$$

$$
D_{x} \phi^{\prime \prime}-D_{s}\left(u_{y}^{\prime}+\phi\right)-D_{a}\left(u_{y}^{\prime}-\phi+2 \psi\right)=0
$$

$$
2 D_{x z} \psi^{\prime \prime}+2 D_{a}\left(u_{y}^{\prime}-\phi+2 \psi\right)=0 .
$$

\section{Analysis of Micropolar-Cosserat elastic panel}

\subsection{In-plane static analysis}

The steps followed for the series of solutions of the equilibrium equations to find out in-plane static responses are elaborated by Anssi T. Karttunen into Appendix A (Karttunen, A. T., Reddy, J. N., \& Romanoff, J. et al. , 2018). The solutions of a static 
81

system is generated by the decoupling of Eqs. (25) to (27) using mathematical tools such as MAPLE. 1-D micropolar-Cosserat elastic panel consists of three displacements and three force vector. Hence, six boundary conditions need to be solved corresponding to the six-state vectors namely, $u_{y}, \phi, \psi, M_{x}, Q_{x y}$ and $P_{x z}$. The displacement equations in the form of constant stiffness parameter are written as

$$
u_{y}=\left[c_{1}-c_{2} x-\frac{1}{2} c_{3} x^{2}+c_{4}\left\{(a-b) x-\frac{x^{3}}{3}\right\}-\alpha\left(c_{5} e^{\beta x}-c_{6} e^{-\beta x}\right)\right],
$$

$$
\phi=\left[c_{2}+c_{3} x+c_{4}\left\{(a+b)+x^{2}\right\}-d\left(c_{5} e^{\beta x}+c_{6} e^{-\beta x}\right)\right],
$$

$$
\psi=\left[c_{2}+c_{3} x+c_{4} x^{2}+\left(c_{5} e^{\beta x}+c_{6} e^{-\beta x}\right)\right],
$$

where

$$
\begin{aligned}
a & =\frac{D_{x}+D_{x z}}{D_{s}}, b=\frac{D_{x z}}{D_{a}}, d=\frac{2 D_{x z}}{D_{x}}, \alpha^{2}=\frac{2 D_{x z}\left[\left(D_{x}+D x z\right) D_{a}+D_{s} D_{x z}\right]^{2}}{D_{x} D_{s} D_{a}\left(D_{x}+2 D_{x z}\right)\left(D_{a}-D_{s}\right)}, \text { and } \\
\beta^{2} & =\frac{2 D_{s} D_{a}\left(D_{x}+2 D_{x z}\right)}{D_{x} D_{x z}\left(D_{a}-D_{s}\right)} .
\end{aligned}
$$

The force equations based on stiffness parameters are derived with the help of stress or force resultants Eqs. (18) to (21) and displacement Eqs. (28) to (30) are as follows

$$
\begin{gathered}
M_{x}=D_{x}\left[c_{3}+2 c_{4} x-d \beta\left(c_{5} e^{\beta x}-c_{6} e^{-\beta x}\right)\right] \\
Q_{x y}=\left[2\left(D_{s} a+D_{a} b\right) c_{4}+\left\{\left(D_{a}-D_{s}\right) \alpha \beta-d\left(D_{a}+D_{s}\right)-2 D_{a}\right\} \ldots\right. \\
\left.\ldots\left(e^{\beta x} c_{5}+e^{-\beta x} c_{6}\right)\right],
\end{gathered}
$$

$$
P_{x z}=2 D_{x z}\left[c_{3}+2 c_{4} x+\beta\left(c_{5} e^{\beta x}-c_{6} e^{-\beta x}\right)\right] .
$$

Substituting $x=0, u_{y}=u_{y_{1}}, \phi=\phi_{1}, \psi=\psi_{1}, M_{x}=M_{x_{1}}, Q_{x y}=Q_{x y_{1}}$ and $P_{x z}=$ $P_{x z_{1}}$ in Eqs. (28) to (33). The matrix relation between the state-vector and coefficients can be expressed as

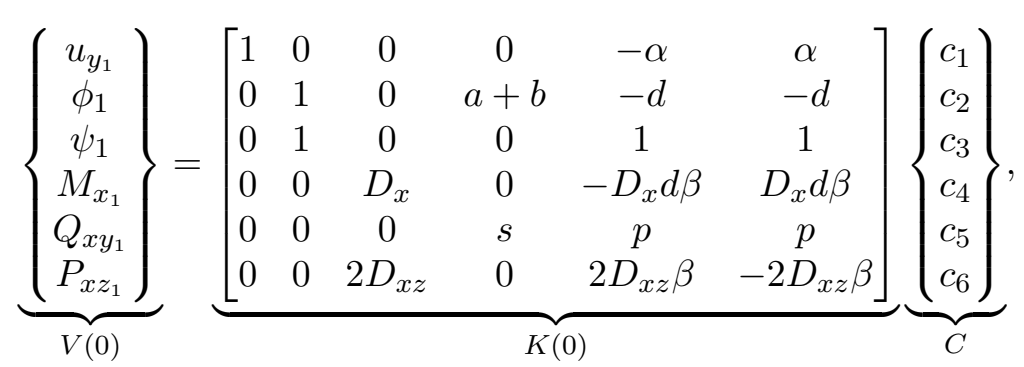


Similarly substituting $x=L, u_{y}=u_{y_{2}}, \phi=\phi_{2}, \psi=\psi_{2}, M_{x}=M_{x_{2}}, Q_{x y}=$ $Q_{x y_{2}}$ and $P_{x z}=P_{x z_{2}}$ in Eqs. (28) to (33). The matrix relation between the statevector and coefficients can be expressed as

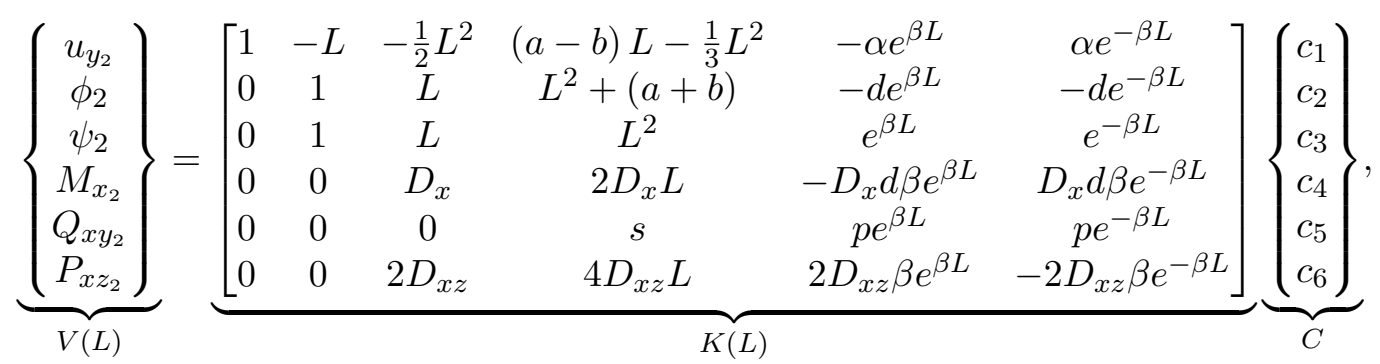

where, $p=\left(D_{a}-D_{s}\right) \alpha \beta-\left(D_{a}+D_{s}\right) d-2 D_{a}$ and $s=2 D_{a} b+2 D_{s} a$. From the Eqs. (34), and (35) we can write

$$
\{C\}_{6 \times 1}=[K(0)]_{6 \times 6}^{-1}\{V(0)\}_{6 \times 1},
$$

$$
\{C\}_{6 \times 1}=[K(L)]_{6 \times 6}^{-1}\{V(L)\}_{6 \times 1} .
$$

By putting the value of the coefficient of Eq. (36) into Eq. (37), the relation between the state vector for two boundary values can be written as

$$
\{V(L)\}_{6 \times 1}=\underbrace{[K(L)]_{6 \times 6}[K(0)]_{6 \times 6}^{-1}}_{T_{s}}\{V(0)\}_{6 \times 1} .
$$

Let us assume, the transfer matrix of a static system

$$
\left[T_{s}\right]_{6 \times 6}=\left[\begin{array}{ll}
T_{11} & T_{12} \\
T_{21} & T_{22}
\end{array}\right]_{6 \times 6} .
$$

293 From the Eq. (38) we can write

$$
\left\{\begin{array}{l}
D_{2} \\
F_{2}
\end{array}\right\}_{6 \times 1}=\left[\begin{array}{ll}
T_{11} & T_{12} \\
T_{21} & T_{22}
\end{array}\right]\left\{\begin{array}{l}
D_{1} \\
F_{1}
\end{array}\right\}_{6 \times 1},
$$

where $\left\{D_{k}\right\}^{T}=\left\{\begin{array}{lll}u_{y_{k}} & \phi_{k} & \psi_{k}\end{array}\right\},\left\{F_{k}\right\}^{T}=\left\{\begin{array}{lll}M_{x_{k}} & Q_{x y_{k}} & P_{x z_{k}}\end{array}\right\}$ and $\mathrm{k}=1,2$. From the Eq. (39), the relationship between displacement, force, and transfer matrix is expressed as

$$
\left\{\begin{array}{c}
u_{y_{2}} \\
\phi_{2} \\
\psi_{2}
\end{array}\right\}=\left[T_{11}\right]_{3 \times 3}\left\{\begin{array}{c}
u_{y_{1}} \\
\phi_{1} \\
\psi_{1}
\end{array}\right\}+\left[T_{12}\right]_{3 \times 3}\left\{\begin{array}{l}
M_{x_{1}} \\
Q_{x y_{1}} \\
P_{x z_{1}}
\end{array}\right\}
$$

$$
\left\{\begin{array}{l}
M_{x_{2}} \\
Q_{x y_{2}} \\
P_{x z_{2}}
\end{array}\right\}=\left[T_{21}\right]_{3 \times 3}\left\{\begin{array}{c}
u_{y_{1}} \\
\phi_{1} \\
\psi_{1}
\end{array}\right\}+\left[T_{22}\right]_{3 \times 3}\left\{\begin{array}{l}
M_{x_{1}} \\
Q_{x y_{1}} \\
P_{x z_{1}}
\end{array}\right\} .
$$


A homogeneous panel is solved as a 1-D cantilever elastic panel. Hence, for fixed end, $\left\{D_{1}\right\}=0$ and for free end, $M_{x_{2}}=0$ but $Q_{x y_{2}} \neq 0$ and $P_{x z_{2}} \neq 0$. It can be derived from Eqs. (40) and (41)

$$
\left\{\begin{array}{c}
u_{y_{2}} \\
\phi_{2} \\
\psi_{2}
\end{array}\right\}=\left[T_{12}\right]_{3 \times 3}\left[T_{22}\right]_{3 \times 3}^{-1}\left\{\begin{array}{c}
0 \\
Q_{x y_{2}} \\
P_{x z_{2}}
\end{array}\right\},
$$

where flexibility and stiffness matrix of cantilever panel are, $[F]_{3 \times 3}=$ $\left[T_{12}\right]_{3 \times 3}\left[T_{22}\right]_{3 \times 3}^{-1}$ and $\left[K_{s}\right]_{3 \times 3}=[F]_{3 \times 3}^{-1}$, respectively. The value of $\left\{D_{2}\right\}^{T}=$ $\frac{1}{L}[F]_{3 \times 3}$, means curvature moment, $P_{x z_{2}}=2 G K_{x z_{2}} l^{2}$. The Eqs. (37) and (42) the value of coefficient matrix is

$$
\{C\}_{6 \times 1}=\underbrace{\left[K_{c}\right]_{6 \times 3}^{-1}\left[T_{22}\right]_{3 \times 3}^{-1}}_{K_{t}}\left\{\begin{array}{c}
0 \\
Q_{x y_{2}} \\
P_{x z_{2}}
\end{array}\right\}_{3 \times 1},
$$

where

$$
\left[K_{t}\right]=\left[\begin{array}{ccc}
-\frac{\alpha}{D_{x} \beta(1+d)} & 0 & \frac{\alpha}{2 D_{x z} \beta(1+d)} \\
0 & -\frac{a+b}{q} & 0 \\
\frac{1}{D_{x}(1+d)} & 0 & \frac{d}{2 D_{x z}(1+d)} \\
0 & \frac{1+d}{q} & 0 \\
-\frac{1}{2 D_{x} \beta(1+d)} & \frac{a+b}{2 q} & \frac{1}{4 D_{x z} \beta(1+d)} \\
\frac{1}{2 D_{x} \beta(1+d)} & \frac{a+b}{2 q} & -\frac{1}{4 D_{x z} \beta(1+d)}
\end{array}\right]_{6 \times 3} \text {, and }
$$

$q=\left(D_{a}-D_{s}\right)\{\alpha \beta(a+b)-d(a-b)-2 a\}$.

Substitute the Eq. (44) into Eq. (43), and upshots of the Eq. (43) is used to find out macro and micro displacements of homogeneous panel via Eqs. (28) to (30). Yield stress and force resultants can be obtained by putting the values of displacements into the Eqs. (18) to (21).

\subsection{Finite Element analysis for static response}

The FE model (Plane-stress element) for the plot of displacements is shown in Fig. 3. The volume and surface area of panel are $L W T$ and $2(L W+L T+W T)$, respectively. The detailed description of FE model are given as

(1) Geometry: 2-D planar deformable shell element.

(2) Section: Homogeneous solid.

(3) Mesh size: $0.025 \mathrm{~m}$.

(4) Mesh controls: Quad-dominated.

(5) Element shape: Quad. 


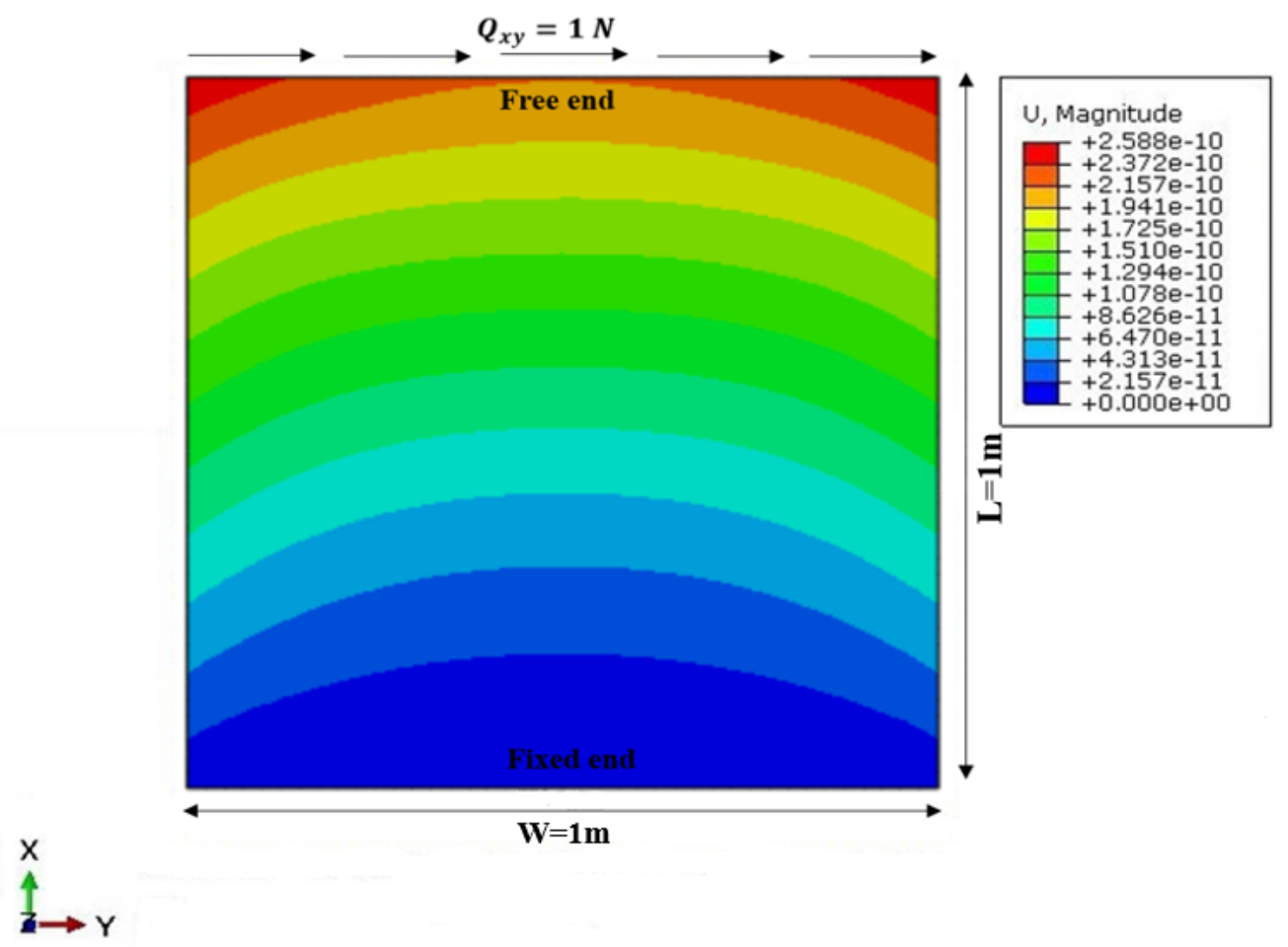

Figure 3. In-plane static displacement (m) due to the surface traction force.

\subsection{Comparative results of static panel}

Consider a homogeneous cantilever panel with geometric and material properties to study the comparative macro and micro-displacements. Modulus of elasticity, $E=2.1 \times 10^{11} \mathrm{~N} / \mathrm{m}^{2}$, Poisson ratio, $\nu=0.30, \rho=7850 \mathrm{~kg} / \mathrm{m}^{3}, L=1$ to $3 \mathrm{~m}$, $W=0.15$ to $2.75 \mathrm{~m}$ and constant $T=0.15 \mathrm{~m}$. The Micropolar-Cosserat analysis, Timoshenko and Goodier exact cantilever analysis (Augarde, C. E., \& Deeks, A. J. et al. , 2008) and Timoshenko couple stress analysis (Asghari, M., Kahrobaiyan, M. H., Rahaeifard, M., \& Ahmadian, M. T. et al. , 2011) with respect to FE analysis at 1 $\mathrm{N} / \mathrm{m}^{2}$ surface traction for the varying dimensions are summarised as follows,

\subsubsection{Lateral displacement and stiffness}

Deflection and stiffness of cantilever panels are found directly from FE analysis. Typical graphs for comparative analysis of lateral deflection and stiffness are shown in Fig. 4 and Fig. 5, respectively.

(1) Timoshenko and Goodier exact cantilever (Augarde, C. E., \& Deeks, A. J. et al. 


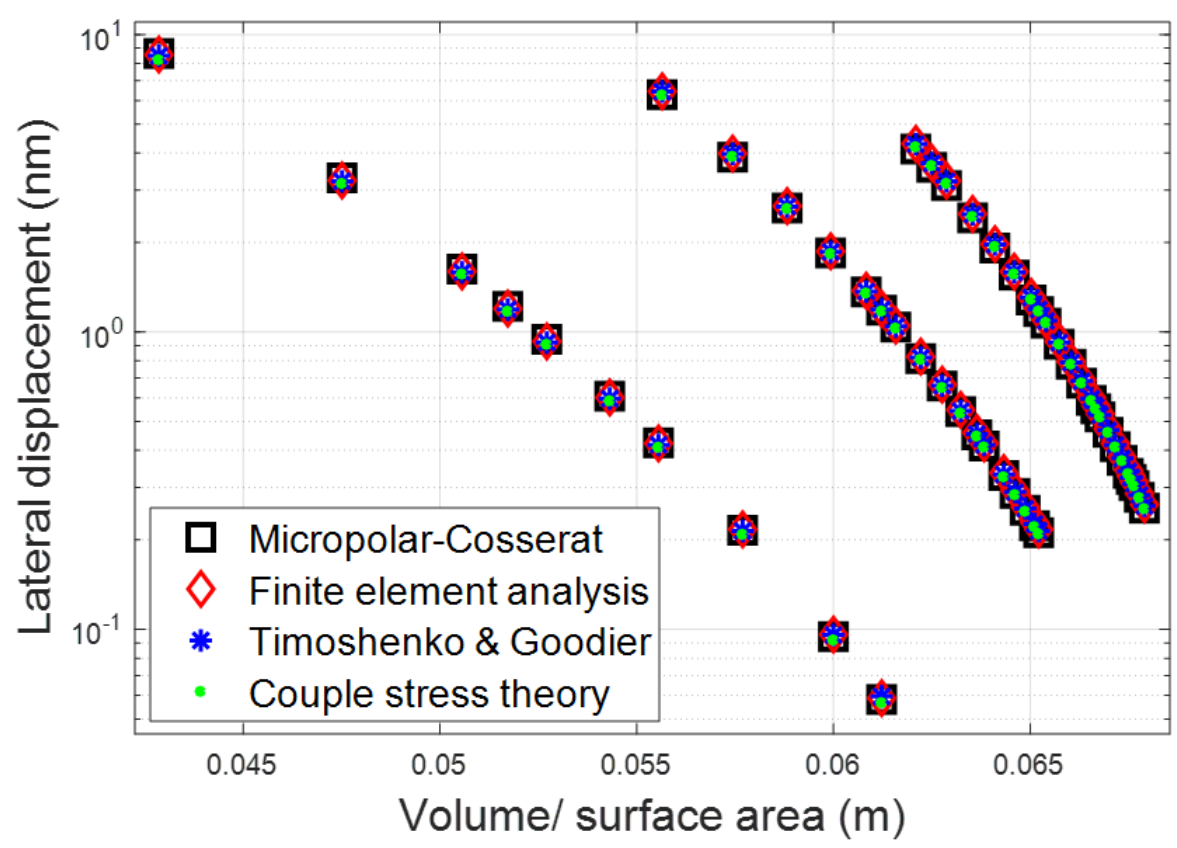

Figure 4. Lateral deflection of the homogeneous panel.



Figure 5. Stiffness of the homogeneous panel.

$$
\begin{array}{r}
u_{x}=\frac{Q_{x y} y}{6 D_{x}}\left[(6 L-3 x) x+(2+\nu)\left(y^{2}-\frac{W^{2}}{4}\right)\right], \\
u_{y}=\frac{Q_{x y}}{6 D_{x}}\left[3 \nu y^{2}(L-x)+(4+5 \nu) \frac{W^{2} x}{4}+(3 L-x) x^{2}\right] .
\end{array}
$$


(2) Timoshenko couple stress (Asghari, M., Kahrobaiyan, M. H., Rahaeifard, M., \& Ahmadian, M. T. et al. , 2011) expression for displacement

$$
\begin{array}{r}
u_{x}=\frac{Q_{x y} y f}{2}\left[(1-e)\left(\frac{\cosh \lambda(x-L)}{\cosh \lambda L}-1\right)-g\left(\frac{x^{2}}{2}-L x\right)\right], \\
u_{y}=\frac{Q_{x y} f}{2}\left[(1+e)\left(x-\frac{\sinh \lambda(x-l)+\sinh \lambda L}{\lambda \cosh \lambda L}\right)+g\left(\frac{L x^{2}}{2}-\frac{x^{3}}{6}\right)\right],
\end{array}
$$

where,

$$
\begin{aligned}
& \lambda=2 \sqrt{D_{s}\left(\frac{1}{D_{x}}+\frac{1}{2 D_{x z}}\right)}, e=\frac{1}{\lambda^{2}}\left(\frac{\lambda^{2} D_{x}-2 D_{s}}{D_{x}+D_{x z}}\right), f=\frac{1}{D_{s}}\left(\frac{D_{x z}+D_{x}}{2 D_{x z}+D_{x}}\right) \text { and, } \\
& g=\left(\frac{2 D_{s}}{D_{x}+D_{x z}}\right) .
\end{aligned}
$$

\subsubsection{Rotation of cross-section}

The rotation of cross-section is derived with the help of longitudinal and lateral deflection of a panel which are found from FE analysis. Typical sketch and graph for comparison of rotation of cross-section is shown in Fig. 6 and Fig. 7, respectively. The rotation of cross-section about the neutral axis is expressed as

$$
\phi=\left[-\frac{u_{x}}{y}+\frac{\sqrt{A B_{x}^{2}+A B_{y}^{2}}}{y}\right],
$$

343 where, $A B_{x}=-y \sin \theta, A B_{y}=y(1-\cos \theta), y=\frac{W}{2}, \psi=$ micro-rotation, and bending slope, $\theta=\frac{\partial u_{y}}{\partial x}$.

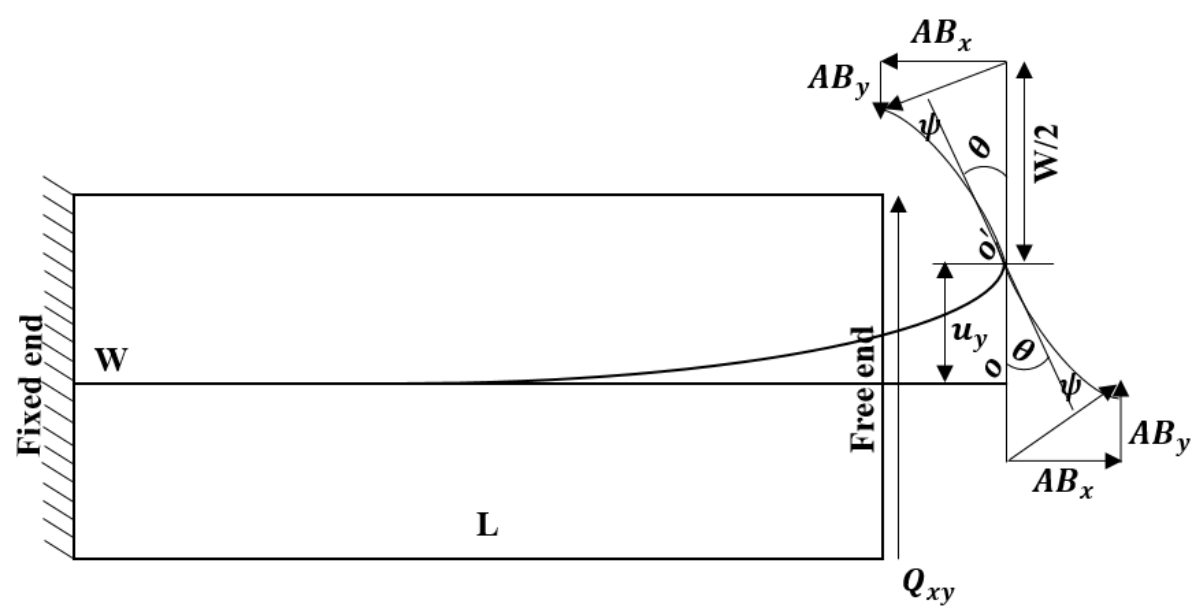

Figure 6. Macro and micro-rotation of a panel. 


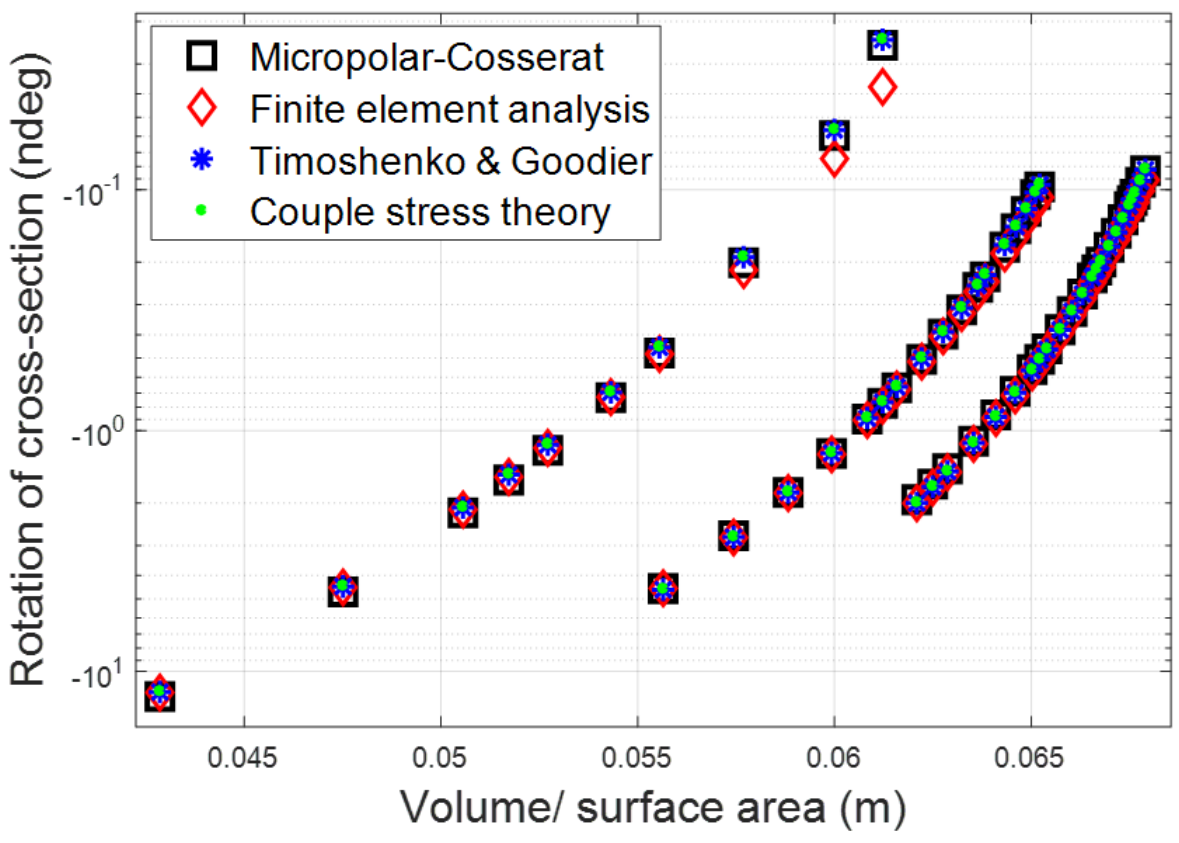

Figure 7. Rotation of the cross-section about the neutral axis.

\subsubsection{Rotation of micro-structure}

The sketch and graph of relative rotation of micro-structure based on the displacement field are shown in Fig. 6 and Fig. 8, respectively. The average rotations of microstructure is

$$
\psi=\frac{1}{2}\left(\phi-\frac{\partial u_{y}}{\partial x}\right)
$$

Using the Eq. (47) into Eq. (48), micro-rotation in based on lateral and longitudinal displacement

$$
\psi=\frac{1}{2}\left[-\frac{u_{x}}{y}+\frac{\sqrt{A B_{x}^{2}+A B_{y}^{2}}}{y}-\frac{\partial u_{y}}{\partial x}\right]
$$

The comparison of FE analysis and Micropolar-Cosserat shows the good agreements with the in-plane static response; macro displacement and micro-rotation concerning the ratio of volume to surface area of the panel. The error appears in the macrodisplacement and micro-rotation is due to imperfection in localization of deformation upon mesh refinement sensitivity. The localization associated with strain softening is neither necessary nor sufficient in setting the constant width of the shear band and energy dissipation during the time of computation (De Borst, R., \& Sluys, L. J. et al. , 1991; Bazant, Z. P., \& Pijaudier-Cabot, G. et al. , 1988; Needleman, A. et al. , 1988). The FE analysis v/s Timoshenko and Goodier's exact solution or Timoshenko couple stress analysis graph also shows the same pattern corresponding to the response. In the case of Timoshenko and Goodier's cantilever, error are caused by the incompatibility 


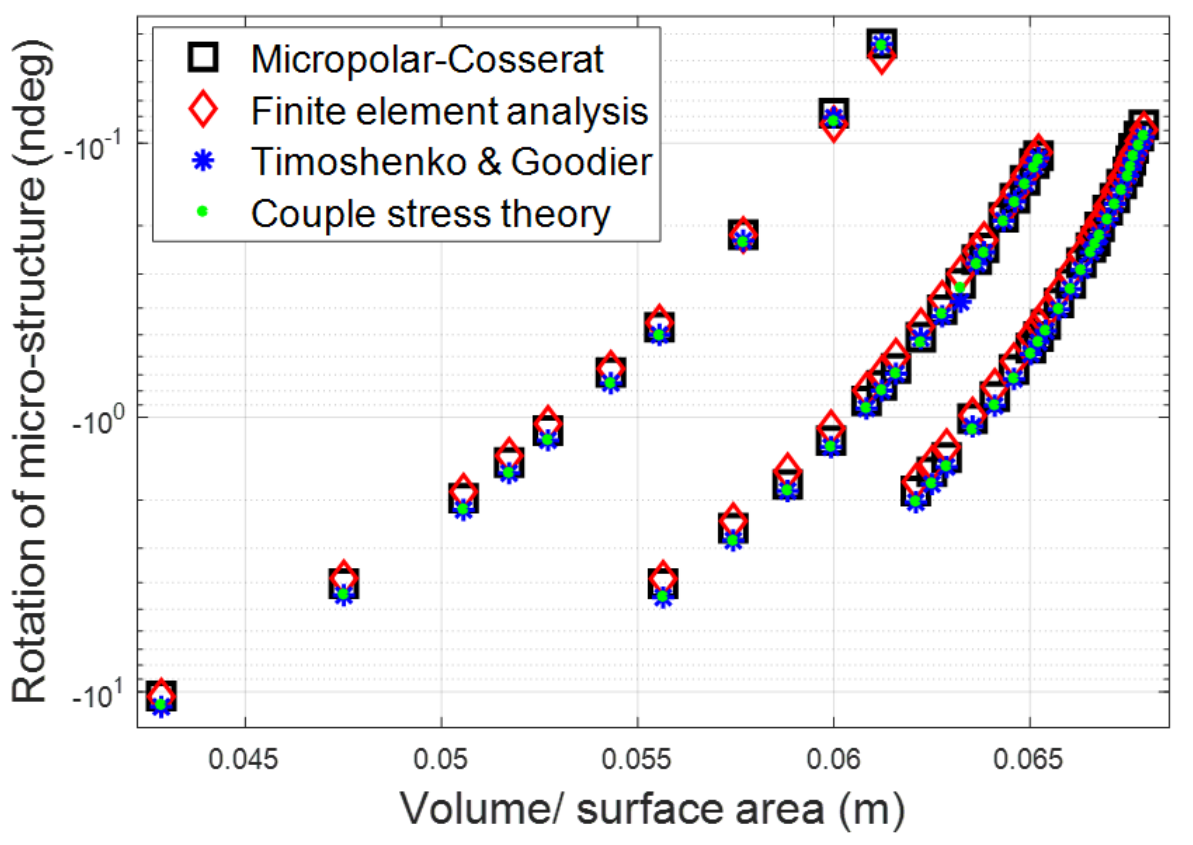

Figure 8. Average micro-rotation of structure based on displacement field of plane-stress element.

between the boundary conditions for complex shear stress at the corners. The top and bottom faces enforce a zero stress boundary condition at the corners, while the applied uniform traction enforces non-zero shear stress boundary conditions at the same places (Augarde, C. E., \& Deeks, A. J. et al. , 2008). In the case of couple stress analysis, this error is due to the asymmetric part of shear stress which does not contribute to energy density into the displacement field of structural system (Asghari, M., Kahrobaiyan, M. H., Rahaeifard, M., \& Ahmadian, M. T. et al. , 2011; Karttunen, A. T., Reddy, J. N., \& Romanoff, J. et al. , 2018).

\subsection{Natural frequencies of the panel}

The dynamic system of coupled Eqs. (22) to (24) have no classical representation. So, It is necessary to represent the coupled system as a two-scale matrix via sufficient and necessary decoupling conditions (Dion, J. M., \& Commault, C. et al. , 1993). The separation variable matrix of coupled equations is expressed as

$$
\underbrace{\left[\begin{array}{ccc}
D_{s}-D_{a} & 0 & 0 \\
0 & D_{x} & 0 \\
0 & 0 & 2 D_{x z}
\end{array}\right]}_{M} \underbrace{\left\{\begin{array}{l}
u_{y}^{\prime \prime} \\
\phi^{\prime \prime} \\
\psi^{\prime \prime}
\end{array}\right\}}_{U^{\prime \prime}}+\underbrace{\left[\begin{array}{ccc}
0 & D_{s}+D_{a} & -2 D_{a} \\
-D_{s}-D_{a} & 0 & 0 \\
2 D_{a} & 0 & 0
\end{array}\right]}_{D} \underbrace{\left\{\begin{array}{c}
u_{y}^{\prime} \\
\phi^{\prime} \\
\psi^{\prime}
\end{array}\right\}}_{U^{\prime}}
$$

$$
+\underbrace{\left[\begin{array}{ccc}
\rho A \omega^{2} & 0 & 0 \\
0 & -\left(D_{s}-D_{a}-\rho I \omega^{2}\right) & -2 D_{a} \\
0 & -2 D_{a} & 4 D_{a}+\rho A J \omega^{2}
\end{array}\right]}_{K_{d}} \underbrace{\left\{\begin{array}{c}
u_{y} \\
\phi \\
\psi
\end{array}\right\}}_{U}=0 .
$$


The generalized formulation of Eq. (50) via representation of state-space method are as follows

$$
M U^{\prime \prime}+D U^{\prime}+K_{d} U=0
$$

373

$$
\begin{gathered}
U^{\prime \prime}+M^{-1} D U^{\prime}+M^{-1} K_{d} U=0, \\
\underbrace{\left\{\begin{array}{l}
U^{\prime \prime} \\
U^{\prime}
\end{array}\right\}_{6 \times 1}}_{X^{\prime}}=\underbrace{\left[\begin{array}{cc}
-M^{-1} D & -M^{-1} K_{d} \\
I_{3} & 0
\end{array}\right]_{6 \times 6}}_{Z} \underbrace{\left\{\begin{array}{l}
U^{\prime} \\
U
\end{array}\right\}_{6 \times 1}}_{X}
\end{gathered}
$$

374

$$
\{X\}^{\prime}=[Z]\{X\}
$$

The solution of the above system of linear differential equations, $\{X\}^{\prime}=[Z]\{X\}$ is $\{X\}=\zeta e^{\Omega x}\{C\}$ or $\{X\}=[S(x)]\{C\}$ (O'neil, P. V. et al. , 2011; Chau, K. T. et al. , 2017). Where, $\zeta$ and $\Omega$ are eigenvector and eigenvalue of $[Z]$, respectively. The solution of a dynamic system is summarised as

$$
\left\{\begin{array}{l}
U^{\prime} \\
U
\end{array}\right\}_{6 \times 1}=\underbrace{\zeta e^{\Omega x}}_{s(x)}\{C\}_{6 \times 1}
$$

375

$$
\left\{\begin{array}{l}
U^{\prime} \\
U
\end{array}\right\}_{6 \times 1}=[S(x)]_{6 \times 6}\{C\}_{6 \times 1} .
$$

The state vector ( or $\mathrm{V}$ matrix) by using displacement $u_{y}, \psi, \phi$ and resultants force Eqs. (18) to (21) can be expressed as

$$
\underbrace{\left\{\begin{array}{c}
u_{y} \\
\phi \\
\psi \\
M_{x} \\
Q_{x y} \\
P_{x z}
\end{array}\right\}}_{V}=\underbrace{\left[\begin{array}{cccccc}
0 & 0 & 0 & 1 & 0 & 0 \\
0 & 0 & 0 & 0 & 1 & 0 \\
0 & 0 & 0 & 0 & 0 & 1 \\
0 & D_{x} & 0 & 0 & 0 & 0 \\
D_{s}+D_{a} & 0 & 0 & 0 & D_{s}-D_{a} & -2 D_{a} \\
0 & 0 & 2 D_{x z} & 0 & 0 & 0
\end{array}\right]}_{R}\left\{\begin{array}{l}
U^{\prime} \\
U
\end{array}\right\}_{6 \times 1},
$$

however, the formulation can be generalized as

$$
\{V\}=[R]\left\{\begin{array}{l}
U^{\prime} \\
U
\end{array}\right\}_{6 \times 1} .
$$

From the Eqs. (53) and (55), the relation between state vector and coefficient is expressed as

$$
\{V(x)\}_{6 \times 1}=[R]_{6 \times 6}\{S(x)\}_{6 \times 6}\{C\}_{6 \times 1} .
$$


The relation between state vector $V_{1}$ and $V_{2}$ by using end conditions, $x=0$ and $x=L$ is expressed as

$$
\{V(L)\}_{6 \times 1}=\underbrace{[R]_{6 \times 6}\{S(L)\}_{6 \times 6}\{S(0)\}_{6 \times 6}^{-1}[R]_{6 \times 6}^{-1}}_{T_{d}}\{V(0)\}_{6 \times 1} .
$$

Let us assume, the transfer matrix of a dynamic system

$$
\left[T_{d}\right]_{6 \times 6}=\left[\begin{array}{ll}
T_{11} & T_{12} \\
T_{21} & T_{22}
\end{array}\right]_{6 \times 6}
$$

so, Eq. (57) can be written as

$$
\{V(L)\}_{6 \times 1}=\left[\begin{array}{ll}
T_{11} & T_{12} \\
T_{21} & T_{22}
\end{array}\right]_{6 \times 6}\{V(0)\}_{6 \times 1} .
$$

The values of forcing frequency (or $\omega$ ) for which the transfer matrix coefficient, $\left[T_{22}\right]_{3 \times 3}$ are zero. Those value are natural frequencies (or $\omega_{n}$ ) of a homogeneous cantilever panel.

\subsection{Finite element analysis for natural frequency}

The FE model (Plane-stress element) for the plot of natural frequencies is shown in Fig. 9. The volume and surface area of panel are $L W T$ and $2(L W+L T+W T)$, respectively. The detailed description of FE model are given as

(1) Geometry: 2-D planar deformable shell element.

(2) Section: Homogeneous solid.

(3) Mesh size: $0.025 \mathrm{~m}$.

(4) Mesh controls: Quad-dominated.

(5) Element shape: Quad.

(6) Element type: CPS8.

\subsection{Comparative analysis of natural frequency}

Consider a homogeneous cantilever panel with geometric and material properties to study the comparative natural frequencies. The young modulus, $E=2.1 \times 10^{11} \mathrm{~N} / \mathrm{m}^{2}$, Poisson ratio, $\nu=0.30, \rho=7850 \mathrm{~kg} / \mathrm{m}^{3}, L=1$ to $3 \mathrm{~m}, W=0.15$ to $2.75 \mathrm{~m}$ and constant $T=0.15 \mathrm{~m}$. The normalised frequencies for Micropolar-Cosserat analysis, Timoshenko (Hutchinson, J. R. et al. , 2001) and Euler (Mukherjee, A. R. I. N. D. A. M., \& Agnivo, G. et al. , 2010) beam theory with respect to the FE analysis for varying dimensions are shown in Fig. 10. The expression of normalized frequency for homogeneous cantilever panel

$$
N_{f}=\omega_{n} \sqrt{\frac{\rho L^{2}}{G}} .
$$




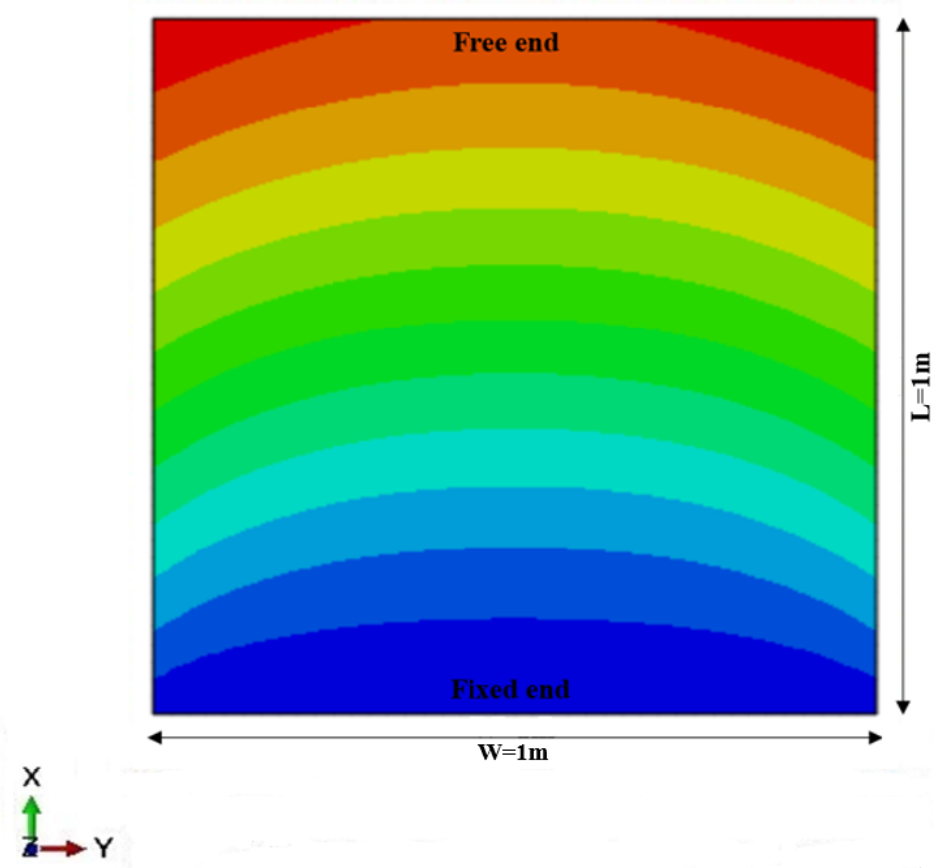

Figure 9. FE model based on static stiffness for the natural frequency of first mode; $533.22 \mathrm{~Hz}$.

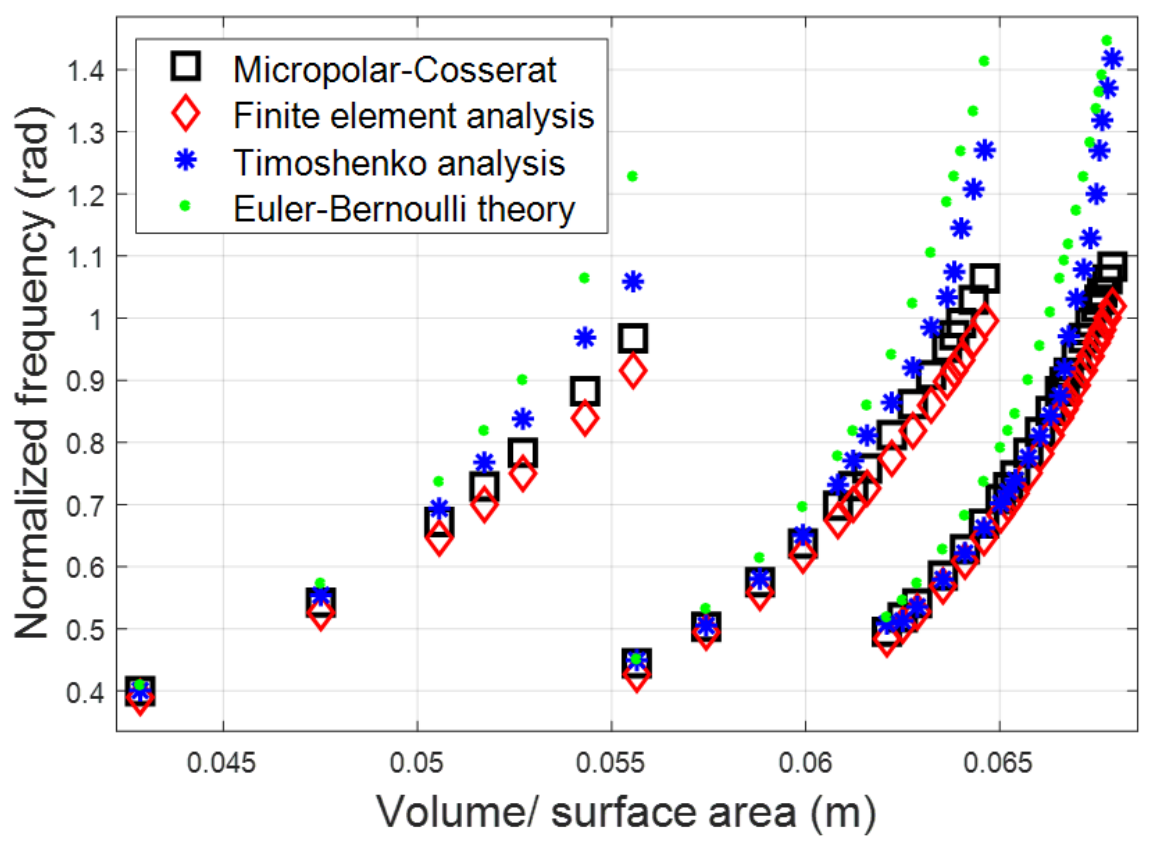

Figure 10. Normalised frequency of the homogeneous panel.

The natural frequency's response of Micropolar-Cosserat theory is very closer to FE analysis with respect to the ratio of volume to surface area of the panels. However, Timoshenko's and Euler's natural frequencies are found to have more differences with respect to FE analysis. This is caused by the existence of micro-rotational waves which 
are not found in classical theories (Singh, D., \& Tomar, S. K. et al. , 2008; Reda, H., Rahali, Y., Ganghoffer, J. F., \& Lakiss, H. et al. , 2016; Karami, B., Shahsavari, D., \& Janghorban, M. et al. , 2018). It is observed that the micro-elastic characteristics are not sufficient for the realistic dispersion of waves. The micro-inertia needed in addition to micro-elastic characteristics (Ramezani, S., Naghdabadi, R., \& Sohrabpour, S. et al. , 2009; Colquitt, D. J., Jones, I. S., Movchan, N. V., \& Movchan, A. B. et al. , 2011; Papargyri-Beskou, S., Polyzos, D., \& Beskos, D. E. et al. , 2009).

\section{Summary of results}

The summary of result from the study of static and dynamic systems based on classical and non-classical theories are as follows:

\subsection{Static system}

- This system is capable to predict the presence of curvature or micro-rotational field of displacement.

- Transfer matrix method is used for the snapshot of the macro and micro displacements of the panels.

- FE analysis of panel and simulations with Timoshenko-coupled stress, Timoshenko and Goodier's exact cantilever, and Micropolar-Cosserat analysis are presented.

- The comparative study shows that differences in macro and micro-deflection and stiffness are up to $3 \%$ if the width of infill walls is limited up to $0.75 \mathrm{~L}$.

\subsection{Dynamic system}

- This system is capable to predict the presence of the dispersive phenomenon of flexural waves.

- The natural frequencies of the panels are evaluated using the transfer matrix approach in conjunction with state-space method. This enables to decouple all the three coupled partial differential equations of motion.

- FE analysis of panel and simulations with Micropolar-Cosserat theory, Timoshenko shear deformation theory, and Euler theory are presented.

- The comparative study shows that differences in natural frequencies are up to $5 \%$ if the width of infill walls is limited up to $0.75 \mathrm{~L}$.

\section{Conclusions}

One-dimensional Micropolar-Cosserat elastic beam theory is used to evaluate the transverse displacements, stiffness, rotation of cross-section, independent rotation of micro-structure, and natural frequencies of the homogeneous panels. The comparison of different theories show that Micropolar-Cosserat theory gives closer result in the case of in-plane static macro-displacement, independent micro-rotation, and natural frequencies with the plane-stress finite element model. Timoshenko and Goodier's exact cantilever analysis, and Timoshenko couple stress analysis also find the best agreement even for higher volume to surface ratio. However, other theories like Timoshenko and Euler-Bernoulli predict the results in acceptable limits only in case of the 
low volume to surface area ratio. The conclusions emphasizes on the contribution of the paper and novelty of this work includes:

- The proposed analytical approach of transfer matrix in this work, can be used to evaluate the static and dynamic response for any type of boundary conditions.

- In the present paper, the curvature moment (or force) has been considered due to asymmetric shear at free end to find the exact in-plane static response.

- The validation of theoretical independent micro-rotation of panel with the help of static response of the plane-stress element is another unique feature of this work.

- The illustration of various beam theories like; Euler-Bernoulli, Timoshenko, Timoshenko and Goodier's exact analysis, Couple stress theory, and their comparison with analytical $\mathrm{v} / \mathrm{s}$ finite elements analysis has not been presented before elsewhere.

- The analytical results evidenced a good agreement with finite element analysis due to incorporation of proposed exact boundary condition at free end.

Acknowledgement $\mathrm{AB}$ acknowledges Inspire faculty grant, grant number: DST/INSPIRE /04/2018/000052. SKS, AB, RV, and SD acknowledge IC Impact grant: DST/INT/CAN/P-03/2019.

Data availability statement The raw/processed data required to reproduce these findings cannot be shared at this time due to technical or time limitations.

\section{References}

Lawson, M. (2001). Light steel framing and modular construction. Steel Technology International, 104-110.

Carrera, E., \& Zozulya, V. V. (2019). Carrera unified formulation (CUF) for the micropolar beams: Analytical solutions. Mechanics of Advanced Materials and Structures, 1-25.

Carrera, E., \& Zozulya, V. V. (2020). Carrera unified formulation (CUF) for the micropolar plates and shells. I. Higher order theory. Mechanics of Advanced Materials and Structures, $1-23$.

Carrera, E., \& Zozulya, V. V. (2020). Carrera unified formulation (CUF) for the micropolar plates and shells. II. Complete linear expansion case. Mechanics of Advanced Materials and Structures, 1-20.

Czekanski, A., \& Zozulya, V. V. (2019). Vibration analysis of nonlocal beams using higherorder theory and comparison with classical models. Mechanics of Advanced Materials and Structures, 1-17.

Wu, B., Pagani, A., Chen, W. Q., \& Carrera, E. (2019). Geometrically nonlinear refined shell theories by Carrera Unified Formulation. Mechanics of Advanced Materials and Structures, $1-21$.

Timoshenko, S. P. (1921). On the additional deflection due to shearing. Glas. Hrvat. Prirodosl. Drus., Zagreb, 33(Part 1, Nr. 1), 50-52.

Ghugal, Y. M., \& Shimpi, R. P. (2001). A review of refined shear deformation theories for isotropic and anisotropic laminated beams. Journal of reinforced plastics and composites, $20(3), 255-272$.

Elishakoff, I., Kaplunov, J., \& Nolde, E. (2015). Celebrating the centenary of Timoshenko's study of effects of shear deformation and rotary inertia. Applied Mechanics Reviews, 67(6).

Xue, Z., Huang, Y., \& Li, M. (2002). Particle size effect in metallic materials: a study by the theory of mechanism-based strain gradient plasticity. Acta Materialia, 50(1), 149-160.

Sun, Z. H., Wang, X. X., Soh, A. K., Wu, H. A., \& Wang, Y. (2007). Bending of nanoscale structures: Inconsistency between atomistic simulation and strain gradient elasticity solu- 
tion. Computational materials science, 40(1), 108-113.

Banerjee, A. (2020). Non-dimensional analysis of the elastic beam having periodic linear spring mass resonators. Meccanica, 1-11.

Asghari, M., Kahrobaiyan, M. H., Rahaeifard, M., \& Ahmadian, M. T. (2011). Investigation of the size effects in Timoshenko beams based on the couple stress theory. Archive of Applied Mechanics, 81(7), 863-874.

Chen, W., \& Si, J. (2013). A model of composite laminated beam based on the global-local theory and new modified couple-stress theory. Composite Structures, 103, 99-107.

Labuschagne, A., van Rensburg, N. J., \& Van der Merwe, A. J. (2009). Comparison of linear beam theories. Mathematical and Computer Modelling, 49(1-2), 20-30.

Chen, W., \& Wang, Y. (2016). A model of composite laminated Reddy plate of the global-local theory based on new modified couple-stress theory. Mechanics of Advanced Materials and Structures, 23(6), 636-651.

Karttunen, A. T., Romanoff, J., \& Reddy, J. N. (2016). Exact microstructure-dependent Timoshenko beam element. International Journal of Mechanical Sciences, 111, 35-42.

Rubin, M. B. et al. (2013). Cosserat theories: shells, rods and points (Vol. 79). Springer Science \& Business Media.

Ebrahimi, F., \& Barati, M. R. (2018a). Vibration analysis of parabolic shear-deformable piezoelectrically actuated nanoscale beams incorporating thermal effects. Mechanics of Advanced Materials and Structures, 25(11), 917-929.

Ebrahimi, F., \& Barati, M. R. (2018b). Longitudinal varying elastic foundation effects on vibration behavior of axially graded nanobeams via nonlocal strain gradient elasticity theory. Mechanics of Advanced Materials and Structures, 25(11), 953-963.

Sobhy, M., \& Zenkour, A. M. (2020). The modified couple stress model for bending of normal deformable viscoelastic nanobeams resting on visco-Pasternak foundations. Mechanics of Advanced Materials and Structures, 27(7), 525-538.

Ventsel, E., Krauthammer, T., \& Carrera, E. J. A. M. R. (2002). Thin plates and shells: theory, analysis, and applications. Appl. Mech. Rev., 55(4), B72-B73.

Toupin, R. A. (1964). Theories of elasticity with couple-stress. BM Watson Research Center Yorktown Heights, New York.

Cosserat, E., \& Cosserat, F. (1909). Théorie des corps déformables. A. Hermann et fils, Paris.

Karttunen, A. T., Reddy, J. N., \& Romanoff, J. (2018). Micropolar modeling approach for periodic sandwich beams. Composite Structures, 185, 656-664.

Noor, A. K., \& Nemeth, M. P. (1980). Micropolar beam models for lattice grids with rigid joints. Computer Methods in Applied Mechanics and Engineering, 21(2), 249-263.

Ramezani, S., Naghdabadi, R., \& Sohrabpour, S. (2009). Analysis of micropolar elastic beams. European Journal of Mechanics-A/Solids, 28(2), 202-208.

Zozulya, V. V. (2018). Higher order theory of micropolar plates and shells. ZAMM-Journal of Applied Mathematics and Mechanics/Zeitschrift für Angewandte Mathematik und Mechanik, 98(6), 886-918.

Mindlin, R. D. (1965). Second gradient of strain and surface-tension in linear elasticity. International Journal of Solids and Structures, 1(4), 417-438.

Nowacki, W. (1972). Theory of micropolar elasticity. Department for Mechanics of Deformable Bodies, (No. 25). Berlin: Springer.

Eringen, A. C. (1968). Mechanics of micromorphic continua. Mechanics of Generalized Continua. E. Kroner (ed.), IUTAM Symposium, Freudenstadt, (pp. 18-35).

Mindlin, R. D., \& Tiersten, H. F. (1962). Effects of couple-stresses in linear elasticity (No. CU-TR-48). Columbia Univ New York.

Nowacki, W. (1974). The linear theory of micropolar elasticity. In Micropolar Elasticity (pp. 1-43). Springer, Vienna.

Eringen, A. C. (1999). Theory of micropolar elasticity. In Microcontinuum field theories (pp. 101-248). Springer, New York, NY.

Eringen, A. C. (2001). Microcontinuum field theories: II. Fluent media (Vol. 2). Springer Science \& Business Media. 
Eringen, A. C. (2012). Microcontinuum field theories: I. Foundations and solids. Springer Science \& Business Media.

Zozulya, V. V. (2017). Couple stress theory of curved rods. 2-D, high order, Timoshenko's and Euler-Bernoulli models. Curved and Layered Structures, 4(1), 119-133.

Kumar, R., \& Ailawalia, P. (2005). Deformation in micropolar cubic crystal due to various sources. International Journal of Solids and Structures, 42(23), 5931-5944.

Gharahi, A., \& Schiavone, P. (2020). Uniqueness of solution for plane deformations of a micropolar elastic solid with surface effects. Continuum Mechanics and Thermodynamics, 32(1), 9-22.

Mindlin, R. D. (1963). Influence of couple-stresses on stress concentrations. Experimental mechanics, 3(1), 1-7.

Khoei, A. R., Yadegari, S., \& Biabanaki, S. O. R. (2010). 3D finite element modeling of shear band localization via the micro-polar Cosserat continuum theory. Computational Materials Science, 49(4), 720-733.

Cao, Y. P., \& Lu, J. (2005). Size-dependent sharp indentation-I: a closed-form expression of the indentation loading curve. Journal of the Mechanics and Physics of Solids, 53(1), 33-48.

Banerjee, J. R. (2001). Dynamic stiffness formulation and free vibration analysis of centrifugally stiffened Timoshenko beams. Journal of Sound and Vibration, 247(1), 97-115.

Dion, J. M., \& Commault, C. (1993). Feedback decoupling of structured systems. IEEE Transactions on Automatic Control, 38(7), 1132-1135.

Hoffman, R. E., \& Ariman, T. (1968). The application of Micropolar mechanics to composites (No. Themis-UND-68-3). Notre Dame Univ Ind Coll of Engineering.

Vasiliev, V. V., Barynin, V. A., \& Rasin, A. F. (2001). Anisogrid lattice structures-survey of development and application. Composite structures, 54(2-3), 361-370.

Reddy, J. N. (2011). Microstructure-dependent couple stress theories of functionally graded beams. Journal of the Mechanics and Physics of Solids, 59(11), 2382-2399.

Park, S. K., \& Gao, X. L. (2008). Micromechanical modeling of honeycomb structures based on a modified couple stress theory. Mechanics of Advanced Materials and Structures, 15(8), 574-593.

Lam, D. C., Yang, F., Chong, A. C. M., Wang, J., \& Tong, P. (2003). Experiments and theory in strain gradient elasticity. Journal of the Mechanics and Physics of Solids, 51(8), 1477-1508.

Reddy, J. N. (2003). Mechanics of laminated composite plates and shells: theory and analysis. CRC press.

De Borst, R., \& Sluys, L. J. (1991). Localisation in a Cosserat continuum under static and dynamic loading conditions. Computer Methods in Applied Mechanics and Engineering, 90(1-3), 805-827.

De Borst, R. E. N. É. (1991). Simulation of strain localization: a reappraisal of the Cosserat continuum. Engineering computations, MCB UP Ltd.

Tran, T. H., Monchiet, V., \& Bonnet, G. (2012). A micromechanics-based approach for the derivation of constitutive elastic coefficients of strain-gradient media. International Journal of Solids and Structures, 49(5), 783-792.

Tordesillas, A., Peters, J. F., \& Gardiner, B. S. (2004). Shear band evolution and accumulated microstructural development in Cosserat media. International journal for numerical and analytical methods in geomechanics, 28(10), 981-1010.

Asghari, M., Rahaeifard, M., Kahrobaiyan, M. H., \& Ahmadian, M. T. (2011). The modified couple stress functionally graded Timoshenko beam formulation. Materials \& Design, 32(3), $1435-1443$.

Hadjesfandiari, A. R., Hajesfandiari, A., Zhang, H., \& Dargush, G. F. (2017). Size-dependent couple stress Timoshenko beam theory. arXiv preprint arXiv:1712.08527.

Khoei, A. R., \& Karimi, K. (2008). An enriched-FEM model for simulation of localization phenomenon in Cosserat continuum theory. Computational Materials Science, 44(2), 733 749 .

Augarde, C. E., \& Deeks, A. J. (2008). The use of Timoshenko's exact solution for a cantilever beam in adaptive analysis. Finite elements in analysis and design, 44(9-10), 595-601. 
Bazant, Z. P., \& Pijaudier-Cabot, G. (1988). Nonlocal continuum damage, localization instability and convergence, 287-293.

Needleman, A. (1988). Material rate dependence and mesh sensitivity in localization problems. Computer methods in applied mechanics and engineering, 67(1), 69-85.

O'neil, P. V. (2011). Advanced engineering mathematics. Cengage learning.

Chau, K. T. (2017). Theory of differential equations in engineering and mechanics. CRC Press.

Hutchinson, J. R. (2001). Shear coefficients for Timoshenko beam theory. J. Appl. Mech., $68(1), 87-92$.

Mukherjee, A. R. I. N. D. A. M., \& Agnivo, G. (2010). Determination of Natural Frequency of Euler's Beams Using Analytical and Finite Element Method. Department of Mechanical Engineering.

Singh, D., \& Tomar, S. K. (2008). Longitudinal waves at a micropolar fluid/solid interface. International Journal of Solids and Structures, 45(1), 225-244.

Reda, H., Rahali, Y., Ganghoffer, J. F., \& Lakiss, H. (2016). Wave propagation in 3D viscoelastic auxetic and textile materials by homogenized continuum micropolar models. Composite Structures, 141, 328-345.

Karami, B., Shahsavari, D., \& Janghorban, M. (2018). Wave propagation analysis in functionally graded (FG) nanoplates under in-plane magnetic field based on nonlocal strain gradient theory and four variable refined plate theory. Mechanics of Advanced Materials and Structures, 25(12), 1047-1057.

Colquitt, D. J., Jones, I. S., Movchan, N. V., \& Movchan, A. B. (2011). Dispersion and localization of elastic waves in materials with microstructure. Proceedings of the Royal Society A: Mathematical, Physical and Engineering Sciences, 467(2134), 2874-2895.

Papargyri-Beskou, S., Polyzos, D., \& Beskos, D. E. (2009). Wave dispersion in gradient elastic solids and structures: a unified treatment. International Journal of Solids and Structures, $46(21), 3751-3759$. 